\title{
Capillary electrophoresis of sialylated oligosaccharides in milk from different species
}

Lucia Monti $^{{ }^{*}}$, Tiziana Maria Piera Cattaneo ${ }^{\mathrm{b}}$, Mario Orlandic, Maria Claudia Curadi ${ }^{\mathrm{c}}$

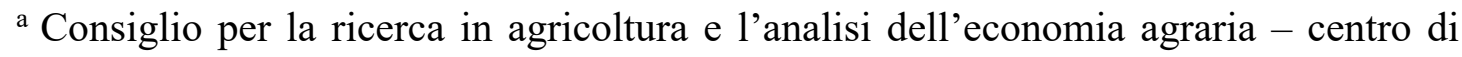
ricerca per le produzioni foraggere e lattiero-casearie (CRA-FLC), Via A. Lombardo 11, 26900 Lodi, Italy

b Consiglio per la ricerca in agricoltura e l'analisi dell'economia agraria, Unità di ricerca per i processi dell'industria agroalimentare (CRA-IAA), Via Venezian, 26, 20133 Milano, Italy

${ }^{\mathrm{c}}$ Dipartimento di Scienze Veterinarie, Università di Pisa, V.le delle Piagge 2, 56124 Pisa, Italy

*Corresponding author. Tel.: +39 037145011; fax: +39 037135579.

E-mail address: lucia.monti@entecra.it (L. Monti)

\begin{abstract}
Oligosaccharides are relevant components of human milk, which have been quite well studied for their pre-biotic effect and their capacity in stimulating the immune system. Since oligosaccharides from milk of non-human mammals received so far less attention, the aim of this work was the application of capillary electrophoresis (CE) for the analysis of sialylated oligosaccharides in cow, goat and equine (mare and donkey) milk to possibly identify potential sources of oligosaccharides to use as health promoting ingredients in functional foods. Human milk was used as reference milk. A recent CE technique was applied to resolve and quantify 3-sialyllactose (3SL), 6-sialyllactose (6-SL) and disialyl-lacto-N-tetraose (DSLNT). Analysis of nonhuman milk samples confirmed differences among species and individuals: DSLNT, which was the most abundant compound in human milk $(455-805 \mu \mathrm{g} / \mathrm{mL})$ was missing in most of the samples. In most cases, 3-SL showed to be the most concentrated of the quantified analytes, with values ranging from 12 to $77 \mu \mathrm{g} / \mathrm{mL}$.
\end{abstract}

Key-words: sialylated oligosaccharides; capillary electrophoresis; mammalian milk. 


\section{Introduction}

Milk is a complex food and the main source of nutrition for infants. Free oligosaccharides (OS) are among the main human milk components, with a concentration variable between 6 and $12 \mathrm{~g} / \mathrm{L}$ in mature milk, and even higher values in colostrum (nearly $24 \mathrm{~g} / \mathrm{L}$ ). On the contrary, in the milk of the more relevant domestic mammals, the OS concentration is 10 to 100 times lower [1, 2]. OS divide into two categories: neutral, often fucosylated at one end, and acidic, having a sialic acid as terminal residual, which gives a negative charge to the molecule [3]. More than 100 different OS have been characterized in human milk [4]; some of these have also been found in the milk of other mammalian species, and they could be used as a source of OS to supplement the diet in case of particular needs [5].

Many different explanations have been advanced concerning the biological roles of individual classes of OS. The main OS in human milk help to protect breast-fed infants from infection, by a pre-biotic action, promoting the selective growth of microorganisms in the gut $[4,6]$. They seem to inhibit the adhesion of pathogenic micro-flora to the intestinal epithelial mucosa, since free OS act as receptor analogs competing with epithelial ligands for bacterial binding [3]. Moreover, there is some evidence that human milk OS influence the glycosylation pattern of epithelial cells, and the immune system [7]. Furthermore, sialylated OS seem to play a role in postnatal brain development, both as a source of galactose for galactocerebroside synthesis, the predominant glycolipid in myelin [3] and as sialic acid supplement for gangliosides and glycoproteins creation in brain's grey matter [8,9].

Anyway, it is difficult to predict a priori the functions a given OS might be mediating, or its relative importance to the organism. At the same time, their biological activity is dependent on individual structural characteristics, and knowledge of OS conformation is very important. The qualitative and quantitative composition of OS in milk of different species is variable [10, 11]. Intra- and interspecies variations are due to genetic reasons and stages of lactation, to suit the requirements of the developing young [1].

The improvement in extraction methods and analytical techniques has revealed more and more a remarkable complexity of these molecules and has cast light on differences and similarities among different species [12].

Analytical methods for quantification and structural analysis of OS include reverseand normal-phase high-performance liquid chromatography [13, 14], high-pH anion 
exchange chromatography and pulsed amperometric detection (HPAEC-PAD) [15], and various mass spectrometry platforms $[16,17,18]$.

In addition, also capillary electrophoresis (CE) has demonstrated to be an interesting technique for the analysis of human milk OS, requiring only simple sample preparation, safe reagents and the use of a regular UV detector [19, 20, 21]. The aim of this work was to test the applicability of a CE procedure for the analysis of 3sialyllactose (3-SL), 6-sialyllactose (6-SL) and disialyl-lacto-N-tetraose (DSLNT) in cow, goat and equine (mare and ass) milk to possibly identify potential sources of oligosaccharides to use as health promoting ingredients in functional foods. Human milk was used as a reference.

\section{Materials and Methods}

\subsection{Standards and Samples}

Commercial crystalline standards of 3-sialyllactose (3-SL), 6-sialyllactose (6-SL) and disialyl-lacto-N-tetraose (DSLNT) (Sigma-Aldrich, Milan, Italy) were used to create dedicated linear calibration curves in the range $39-2500 \mu \mathrm{g} / \mathrm{mL}$. Each standard was diluted in $30 \%$ methanol (Sigma-Aldrich) to obtain the most concentrated standard solution at $2500 \mu \mathrm{g} / \mathrm{mL}$, then diluted again and progressively for the preparation of the $1250,625,156$, and $39 \mu \mathrm{g} / \mathrm{mL}$ standard solutions. Individual milk samples from different species (mare, donkey and human milk) and two bulk milks (cow and goat milk) were stored at $-20^{\circ} \pm 1^{\circ} \mathrm{C}$ immediately after collection, then thawed overnight at $4^{\circ} \pm 1^{\circ} \mathrm{C}$ before analysis. Samples were prepared according to Bao et al. [20]. Briefly, aliquots of $550 \mu \mathrm{L}$ were defatted by centrifugation at $4000 * \mathrm{~g}$ for $15 \mathrm{~min}$ (microcentrifugette ALC 4214). Defatted milk (400 $\mu \mathrm{L}$ ) was mixed with $800 \mu \mathrm{L}$ of pure ethanol (Sigma-Aldrich) and stored overnight at $4^{\circ}+1^{\circ} \mathrm{C}$. Protein precipitate was removed by centrifugation and the clear supernatant, mainly containing oligosaccharides and lactose, was evaporated to dryness under $\mathrm{N}_{2}$ at room temperature. The dried extracts were solubilised by adding $100 \mu \mathrm{L}$ of $30 \%$ methanol and immediately analysed or stored at $-20^{\circ} \pm 1^{\circ} \mathrm{C}$ until $\mathrm{CE}$ analysis.

\subsection{Capillary Electrophoresis}

CE analyses were carried out according to Bao et al. [20]. A P/ACE MDQ Glycoprotein System (Beckman Coulter, Brea, CA, USA), with an uncoated capillary (ID $=50 \mu \mathrm{m}$; effective length $=50 \mathrm{~cm}$; Beckman Coulter, Brea, CA, USA) was used. The run buffer solution was $55 \%$ aqueous $\left(200 \mathrm{mM} \mathrm{NaH}_{2} \mathrm{PO}_{4}, \mathrm{pH} 7.05\right.$, containing 
$100 \mathrm{mM}$ SDS) and 45\% methanol. Sample injection was performed by pressure at 0.7 psi for $6 \mathrm{~s}$ and electrophoretic conditions were positive to negative $(+30 \mathrm{kV})$ at $25^{\circ} \mathrm{C}$. Detection wavelength was $200 \mathrm{~nm}$.

\section{Results and Discussion}

The three sialylated OS which were selected, 3-sialyllactose (3-SL), 6-sialyllactose (6-SL) and disialyl-lacto-N-tetraose (DSLNT), are some of the major human milk oligosaccharides for which commercial standards exist and can be easily found on the market. The electrophoretic procedure applied for their separation showed high peak resolution. The three considered OS, showed specific retention times and separated in less than one hour. Calibration curves for the each standard solution set showed good linearity and good determination coefficients $\left(\mathrm{R}^{2}>0.99\right)$.

Based on these results, the same CE procedure was applied to real milk samples from different species in order to evaluate both the presence of OS and their relative concentrations.

\section{Insert Figure 1}

Examples of the electropherograms of both standards and samples are reported in Figure 1: the electrophoretic pattern of human and equine milk (donkey and mare milk) is shown in comparison with 3-SL, 6-SL and DSLNT standards. A good separation of the three main OS was also guaranteed in real milk samples. In some cases the migration time of the different OS in real samples was slightly faster than in standard solutions: this can be explained by matrix differences influencing the retention time. The correct identification of the three OS was proven by carrying out supplementary electrophoretic runs, adding each standard to real samples, and the assignment was confirmed (data not shown).

Table 1 shows the quantitative results of sialylated OS detected in milk samples.

\section{Insert Table 1}

Analysis of human milk confirmed a significant presence of the three identified OS with some differences in quantity according to donor. Urashima et al. [4] indicated DSLNT, 6-SL and 3-SL among the most predominant acidic OS in many studies. They also highlighted that the concentration of each compound varied between studies, due to the quantification methods used, the donor's ethnicity and the lactation 
stage at which the milk samples were obtained. Anyway, Bode [11] evidenced that sialylated human milk OS contribute to the majority of sialic acid in human milk, an essential nutrient for brain development and cognition. He reported that sialic acid concentrations are significantly higher in the brains of breast-fed infants, compared with formula-fed ones.

Since bovine milk is a common ingredient in infant formula and a common ingredient in human diet, OS content was studied also in that contest. Studies on bovine milk carbohydrates have recently deepened [1], but, it was confirmed that the oligosaccharide composition of bovine milk is simple in comparison to human breast milk. The cow milk sample we analysed showed one of the lowest concentrations of OS, both quantitatively and qualitatively, if compared to the other samples. Only 3-SL was detected at $27.9 \mu \mathrm{g} / \mathrm{mL}$. Tao et al. [22] studied variations in bovine milk OS during early and middle lactation stages: he found that bovine milk contains significantly less free OS compared with human milk, and that sialylated OS represent about $70 \%$ of the total OS. The predominant peak they found in colostrum chromatogram was 3-SL, which concentration rapidly decreased during the first $24 \mathrm{~h}$ of lactation. Similar results were obtained by Nakamura et al. [23].

Among analysed samples, goat milk gave results similar to cow milk. Only 3-SL was detected at a slightly higher concentration. Few previous studies exist on goat milk. Oliveira et al. [24] applied a method similar to our procedure and found the presence of 3-SL in caprine whey, although they did not quantify it. Others researchers $[2,25$, 26] analysed goat milk and found the presence of both 3-SL and 6-SL. In addition, Albrecht et al. [2] evidenced that caprine sialyl oligosaccharides contain Neu5Gc as well as Neu5Ac, with even higher proportions of 3- and 6-glycolyl-lactose (3-GL and 6-GL). Claps et al. [25] also found high values of disialyllactose and evidenced significant differences between two analysed goat breeds and the effect of sampling time.

Results on equine milk were very interesting. To our knowledge, no reference data exist on donkey milk: our values showed in most cases the presence of the same compounds as in human milk, but in less amounts. Donkey milk is usually considered to be a good substitute of human milk, especially for babies suffering from multiple food intollerance who cannot be breast-fed. Its OS profile seems to confirm once more its safe and valid use as infant food. The constant presence of 3-SL 
characterized mare milk samples, while 6-SL and DSLNT were found just in few samples and at very low concentrations. Some researchers studied horse colostrum and found three neutral OS $[27,28]$ and 3-SL as the major acidic component, along with Gal( $\beta 1-4)$ GlcNAc $\alpha 1-p h o s p h a t e ~[29]$, an unusual OS that so far has been found only in the milk/colostrum of cow [1]. In mare colostrum 3-SL was identified as preponderant component also by Nakamura et al. [30] and Albrecht et al. [2]. 6-SL was evidenced, too, although in minor proportion. It is known that colostrum secreted in the first few days postparturition has a composition quite different from that of mature milk. Normally, colostrum contains many biologically active molecules, to provide nutrition and protection to the newborn, but its composition quickly changes. No reference data on OS in mare milk are available, but we could suppose that 3-SL and 6-SL are present at higher concentrations in colostrum and decline rapidly to lower values in milk, as we detected

From our results, it seems that milk from monogastric animals contained more OS than ruminants' milk; at the same time, high OS variability inside the single milk specie was observed, due probably to the stage of lactation and other individual factors.

\section{Conclusions}

The CE procedure applied in this preliminary study confirmed its suitability for the detection, the identification, and the quantification of selected syalilated OS in milk samples from different species, with satisfactory results. The calculated amounts confirmed the high variability of these compounds in milk from different species, according to stage of lactation and individual factors. Further studies need to be developed in order to suggest some milk species as potential sources of oligosaccharides to use as health promoting ingredients in functional foods. Anyway, mare milk seemed to be a very promising font of OS, and its study should be deepened.

\section{References}

[1] T. Urashima, E. Taufik, K. Fukuda, S. Asakuma, Recent advances in studies on milk oligosaccharides of cows and other domestic farm animals, Biosci. Biotechnol. Biochem. 77 (2013) 455-466. 
[2] S. Albrecht, J.A. Lane, K. Mariño, K.A. Al Busadah, S.D. Carrington, R.M. Hickey, P.M. Rudd, A comparative study of free oligosaccharides in the milk of domestic animals, Br. J. Nutr. 111 (2014) 1313-1328.

[3] C. Kunz, S. Rudloff, W. Baier, N. Klein, S. Strobel, Oligosaccharides in human milk: structural, functional, and metabolic aspects, Annu. Rev. Nutr. 20 (2000) 699722.

[4] T. Urashima, M. Kitaoka, T. Terabayashi, K. Fukuda, M. Ohnishi, A. Kobata, Milk Oligosaccharides. In: Oligosaccharides: sources, properties and applications. Ed. N.S. Gordon. Nova Science Publisher, Inc. (NY, USA) 2011.

[5] T. Urashima, E. Taufik, Oligosaccharides in Milk: Their Benefits and Future Utilization. Media Peternakan, 33 (2010) 189-197.

[6] D. Barile, R.A. Rastal, Human milk and related oligosaccharides as prebiotics, Curr. Opin. . Biotechnol. 24 (2013) 214-219.

[7] C. Kunz, S. Kuntz, S. Rudloff, Bioactivity of Human Milk Oligosaccharides. In: "Food Oligosaccharides: Production, Analysis and Bioactivity", First Edition. Ed. F. Javier Moreno and Dr. M. L. Sanz by JohnWiley \& Sons, Ltd 2014.

[8] B. Wang, Sialic Acid Is an Essential Nutrient for Brain Development and Cognition, Annu. Rev. Nutr 29 (2009) 177-222.

[9] S.J.M. ten Bruggencate, I.M.J. Bovee-Oudenhoven, A.L. Feitsma, E. van Hoffen, M.H.C. Schoterman, Functional role and mechanisms of sialyllactose and other sialylated milk oligosaccharides, Nutr Rev 72 (2014) 377-389.

[10] T. Urashima, T. Saito, T. Nakamura, M. Messer, Oligosaccharides of milk and colostrum in non-human mammals, Glycoconj. J. 18 (2001) 357-371.

[11] L. Bode, (2012) Human milk oligosaccharides: Every baby needs a sugar mama, Glycobiology 22 (9) 1147-1162.

[12] R. Mehra, P. Kelly, Milk oligosaccharides: Structural and technological aspects, Int. Dairy J. 16 (2006) 1334-1340.

[13] P. Chaturvedi, C.D. Warren, G.M. Ruiz-Palacios, L.K. Pickering, D.S. Newburg, Milk oligosaccharide profiles by reversed-phase HPLC of their perbenzoylated derivatives, Anal. Biochem. 251 (1997) 89-97.

[14] J. Charlwood, D. Tolson, M. Dwek, P.A. Camilleri, Detailed analysis of neutral and acidic carbohydrates in human milk, Anal. Biochem. 273 (1999) 261-277. 
[15] S. Thurl, B. Muller-Werner, G. Sawatzki, Quantification of individual oligosaccharide compounds from human milk using high-pH anion-exchange chromatography, Anal. Biochem. 235 (1996) 202-206.

[16] J. Zaia, Mass spectrometry of oligosaccharides, Mass Spectrom. Rev. 23 (2004) $161-227$.

[17] M.R. Niñonuevo C.B. Lebrilla, Mass spectrometric methods for analysis of oligosaccharides in human milk, Nutrition Reviews 67 (Suppl. 2) (2009) S216-S226.

[18] L.R. Ruhaak, C.B. Lebrilla, Analysis and role of oligosaccharides in milk, BMB Rep. (2012) 442-451.

[19] Z.J. Shen, C.D. Warren, D.S. Newburg, High-performance capillary electrophoresis of sialylated oligosaccharides of human milk, Anal. Biochem. 279 (2000) 37-45.

[20] Y. Bao, L. Zhu, D.S. Newburg, Quantification of sialyloligosaccharides from human milk by capillary electrophoresis, Anal. Biochem. 370 (2007) 206-214.

[21] Y. Bao, D.S. Newburg Capillary electrophoresis of acidic oligosaccharides from human milk, Electrophoresis 29 (2008) 2508-2515.

[22] N. Tao, E.J. DePeters, J.B. German, R. Grimm, C.B. Lebrilla, Variations in bovine milk oligosaccharides during early and middle lactation stages analyzed by high-performance liquid chromatography-chip/mass spectrometry, J. Dairy Sci. 92 (2009) 2991-3001.

[23] T. Nakamura, H. Kawase, K. Kimura, Y. Watanabe, M. Ohtani, I. Arai, T. Urashima, Concentrations of Sialyloligosaccharides in Bovine Colostrum and Milk during the Prepartum and Early Lactation, J. Dairy Sci. 86 (2003) 1315-1320.

[24] D.L. Oliveira, R.A. Wilbey, A.S. Grandison, L.C. Duarte, L.B. Roseiro, Separation of oligosaccharides from caprine milk whey, prior to prebiotic evaluation, Int. Dairy J. 24 (2012) 102-106.

[25] S. Claps, M.A. Di Napoli, L. Sepe, A.R. Caputo, D. Rufrano, A. Di Trana, G. Annicchiarico, V. Fedele, Sialyloligosaccharides content in colostrum and milk of two goat breeds, Small Ruminant Res. 121 (2014) 116-119.

[26] A. Martinez-Fereza, S. Rudloff, A. Guadix, C.A. Henkel, G. Pohlentz, J.J. Boza, E.M. Guadix, C. Kunz, Goats' milk as a natural source of lactose-derived oligosaccharides: Isolation by membrane technology, Int. Dairy J. 16 (2006) 173-181. 
[27] T. Urashima, T. Sakamoto, H. Ariga, T. Saito, Structure determination of three neutral oligosaccharides obtained from horse colostrum, Carbohydr. Res., 194 (1989) 280-287.

[28] T. Urashima, T. Saito, T. Kimura, Chemical structures of three neutral oligosaccharides obtained from horse (thoroughbred) colostrum, Comp. Biochem. Physiol. B, 100 (1991) 177-83.

[29] Nakamura, T., Amikawa S., Harada T., Saito T., Araia I., Urashima T. (2001) Occurrence of an unusual phosphorylated N-acetyllactosamine in horse colostrum. Biochim. Biophys. Acta 1525, (1-2) 13-18.

[30] T. Nakamura, T. Urashima, The milk oligosaccharides of domestic farm animals, Trends Glycosci. Glyc., 16 (2004) 135-142.

\section{Figure captions:}

Fig. 1 - Electropherograms of OS standard solutions and samples (See Materials and Methods for CE conditions). a) standard solution containing 6-SL (1), 3-SL (2), DSLNT (3); b) an individual donkey milk sample; c) an individual mare milk sample; d) an individual human milk sample.

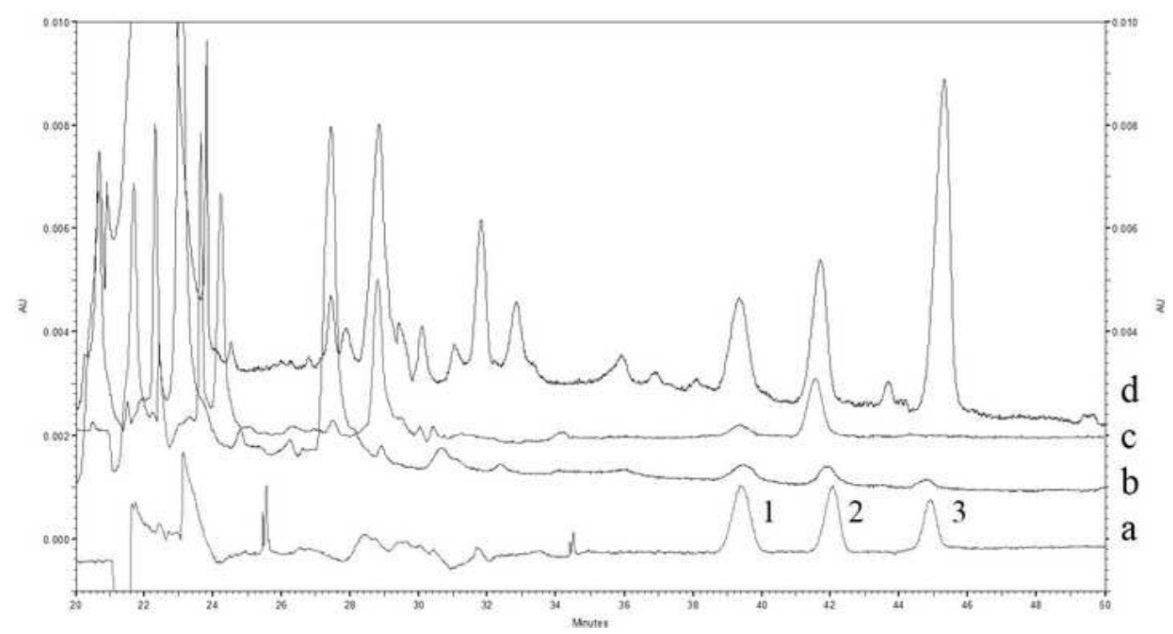




\section{Table 1}

Content of sialylated oligosaccharides in samples of milk from different species; values are expressed in $\mu \mathrm{g} / \mathrm{mL}$; (3-SL: 3-sialyllactose; 6-SL: 6-sialyllactose; DSLNT: disialyl-lacto-N-tetraose); n.d.: not detectable.

\begin{tabular}{|c|c|c|c|c|}
\hline Species & Samples & 3-SL & 6-SL & DSLNT \\
\hline \multirow{3}{*}{ Donkey } & 1 & 16.9 & 12.9 & 11.8 \\
\hline & 2 & 14.3 & 23.3 & 5.0 \\
\hline & 3 & 12.4 & 20.0 & n.d. \\
\hline \multirow{3}{*}{ Mare } & 1 & 77.2 & 0.9 & 3.8 \\
\hline & 2 & 55.7 & 0.4 & n.d. \\
\hline & 3 & 72.5 & 2.6 & n.d. \\
\hline \multirow{2}{*}{ Human } & 1 & 196.3 & 98.0 & 455.5 \\
\hline & 2 & 839.6 & 46.4 & 805.7 \\
\hline Goat & & 67.4 & n.d. & n.d. \\
\hline Cow & & 27.9 & n.d. & n.d. \\
\hline
\end{tabular}

